\title{
Digit derivatives and application to zeta measures
}

\author{
by \\ SAngtae JeOng (Incheon)
}

1. Introduction. Let $\mathbf{k}=\mathbb{F}_{q}(T)$ be a rational function field over a finite field $\mathbb{F}_{q}$ of $q$ elements where $q$ is a power of a prime $p$ and let $\mathbf{k}_{\infty}=\mathbb{F}_{q}((\pi))$ be the completion of $\mathbf{k}$ at the infinite place $\pi=1 / T$. Then we denote by $\mathbf{A}=\mathbb{F}_{q}[T], \mathbf{A}_{\infty}=\mathbb{F}_{q}[[\pi]]$ the rings of integers of $\mathbf{k}, \mathbf{k}_{\infty}$ respectively and by $\mathbf{C}_{\infty}$ the completion of an algebraic closure of $\mathbf{k}_{\infty}$. Throughout, we also denote by $\mathbf{A}^{+}$the set of monic polynomials in $T$ of $\mathbf{A}$, which corresponds to the set $\mathbb{Z}^{+}$of positive integers. We then have well known analogies with the classical case: $\mathbb{Z} \leftrightarrow \mathbf{A}, \mathbb{Q} \leftrightarrow \mathbf{k}, \mathbb{R} \leftrightarrow \mathbf{k}_{\infty}, \mathbb{C} \leftrightarrow \mathbf{C}_{\infty}$. Over $\mathbf{A}$, Carlitz [C1] first studied the special values of an analogue of the classical Riemann zeta function, defined as the power sums

$$
\zeta(k)=\sum_{a \in \mathbf{A}^{+}} \frac{1}{a^{k}} \quad \text { for } k \in \mathbb{Z}^{+} .
$$

In connection with arithmetic and analytic theory over function fields the zeta function is more generally extended by Goss [Go2] into the character space $S_{\infty}=\mathbf{C}_{\infty}^{\times} \times \mathbb{Z}_{p}$, where $\mathbb{Z}_{p}$ is the ring of $p$-adic integers. In particular, over $\mathbf{A}$ the zeta function $\zeta(s)=\zeta\left(s_{1}, s_{2}\right)$ with $s=\left(s_{1}, s_{2}\right) \in S_{\infty}$ is defined as

$$
\zeta(s)=\sum_{a \in \mathbf{A}^{+}} \frac{1}{a^{s}}
$$

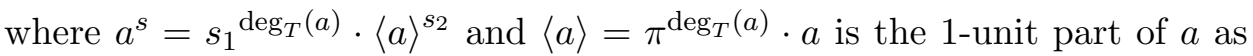
an element in $\mathbf{k}_{\infty}^{\times}$. It is then easily seen that $\zeta\left(T^{k}, k\right)=\zeta(k)$, so Goss's zeta function coincides with that of Carlitz for $k \geq 1$.

The zeta measures, which are defined below, involve both the special values $\zeta(x,-k)$ for non-negative integers $k$ and orthonormal bases for the space of continuous functions defined on the integer ring of the completion of $\mathbf{k}$ at any place. Let $v$ be a finite place of $\mathbf{k}$, so $v$ corresponds to a prime

2000 Mathematics Subject Classification: Primary 11M38.

This work was supported by Korea Research Foundation Grant, KRF-2002-003C00002. 
ideal of $\mathbf{A}$ generated by a monic irreducible polynomial $\pi_{v}$ of $\mathbf{A}$. Let $\mathbf{A}_{v}$ be the completion of $\mathbf{A}$ at $v, \mathbf{k}_{v}$ be the fraction field of $\mathbf{A}_{v}$, and let $C\left(\mathbf{A}_{v}, \mathbf{k}_{v}\right)$ be the $\mathbf{k}_{v}$-Banach space of continuous functions from $\mathbf{A}_{v}$ into $\mathbf{k}_{v}$ equipped with the usual sup norm. It is then well known (see [W, Go1, Co, Sn, J2]) that the space $C\left(\mathbf{A}_{v}, \mathbf{k}_{v}\right)$ has two sets of orthonormal bases consisting of the Carlitz polynomials and digit derivatives. Now the zeta measure is defined as a 1-parameter family of measures for all $x$ such that $|x|_{\infty}<1$. Indeed, the zeta measure $\mu_{x}$ on $\mathbf{A}_{v}$ is given by an integral of the form

$$
\int_{\mathbf{A}_{v}} t^{k} d \mu_{x}(t)=z(x,-k)
$$

for integers $k \geq 0$, where $z(x,-k)=\zeta\left(T^{-k} x,-k\right)$.

Similarly the zeta measure $\mu_{x}^{(\infty)}$ on $\mathbf{A}_{\infty}$ is given by

$$
\int_{\mathbf{A}_{\infty}} t^{k} d \mu_{x}^{(\infty)}(t)=\zeta(x,-k) .
$$

By an isomorphism between the algebra of measures with the usual convolution product and the algebra of formal divided power series ([Sn, Go2]) each measure $\mu_{x}$ is mapped uniquely to a divided power series $f_{\mu_{x}}(z)=$ $\sum_{k=0}^{\infty} m_{x}(k) \frac{z^{k}}{k !}$, where $m_{x}(k)=\int_{\mathbf{A}_{v}} f_{k} d \mu_{x}$ for an orthonormal basis $\left\{f_{k}\right\}_{k \geq 0}$ of $C\left(\mathbf{A}_{v}, \mathbf{k}_{v}\right)$ coming from an orthonormal basis of the $\mathbb{F}_{q}$-linear functions via the digit expansion principle as in [Co]. From this we see that the divided power series associated to the zeta measures depend on the choice of orthonormal bases for the space $C\left(\mathbf{A}_{v}, \mathbf{k}_{v}\right)$.

An explicit computation of the zeta measure at a finite place of $\mathbf{A}$ with respect to the Carlitz polynomials was done completely by D. Thakur [Th]. Motivated by Thakur's work, recently Z. Yang [Y2] completed an extensive computation of the divided power series associated to the zeta measure at the infinite place with the counterpart of the Carlitz polynomials for the parameter $\pi$. As for the digit derivatives B. Snyder [Sn] calculated the zeta measure only at places of degree 1 in $\mathbf{A}$ via a highly complicated computation.

In this paper we derive some interesting interpolation results on $\mathbf{A}$ (hence on $\left.\mathbb{F}_{q}[[T]]\right)$ for the digit derivatives, which are parallel to those known for the Carlitz polynomials in [C2]. We then give a simple alternative proof of Snyder's result by relating interpolation to the zeta measure as in [Th]. In addition, we explicitly compute the divided power series associated to the zeta measure at $\mathbf{A}_{\infty}$ in terms of the counterpart of digit derivatives for the parameter $\pi$. The latter work is closely patterned on Yang's computation [Y1, Y2]. For it can be observed throughout Section 2 (see also [C2, J1]) that the Carlitz polynomials and digit derivatives share many properties of orthonormal bases of $C\left(\mathbb{F}_{q}[[T]], \mathbb{F}_{q}((T))\right)$. 
2. Properties of digit derivatives. For each $n \geq 0$ the $n$th hyperdifferential operator $\mathcal{D}_{n}$ is defined by

$$
\mathcal{D}_{n}\left(T^{m}\right)=\left(\begin{array}{c}
m \\
n
\end{array}\right) T^{m-n} \quad \text { for } m \geq 0
$$

and is extended to the polynomial ring $\mathbf{A}=\mathbb{F}_{q}[T]$ by $\mathbb{F}_{q}$-linearity. It is also well known that the operator $\mathcal{D}_{n}$ on $\mathbf{A}$ can be extended uniquely to $\mathbf{k}$ and then continuously to the completion of $\mathbf{A}$ at any place. The readers are referred to $[\mathrm{H}, \mathrm{HS}, \mathrm{T}, \mathrm{Co}]$ for more properties of $\mathcal{D}_{n}$. In particular, at the place $(T)$ the set of $\mathcal{D}_{n}$ forms an orthonormal basis of the space of continuous $\mathbb{F}_{q}$-linear functions from $\mathbb{F}_{q}[[T]]=\mathbf{A}_{T}$ to $\mathbb{F}_{q}((T))=\mathbf{k}_{T}$, denoted $L C\left(\mathbb{F}_{q}[[T]], \mathbb{F}_{q}((T))\right)$, which is independently proved by B. Snyder, the author and K. Conrad ([Sn, J2, Co]).

With a basis of $L C\left(\mathbb{F}_{q}[[T]], \mathbb{F}_{q}((T))\right)$, one can easily construct an orthonormal basis of the entire space $C\left(\mathbb{F}_{q}[[T]], \mathbb{F}_{q}((T))\right)$ by the digit principle of K. Conrad [Co]. More precisely, we form the $q$-adic extensions of $\mathcal{D}_{n}$, called digit derivatives, as follows. For an integer $k \geq 0$ written in base $q$ as

$$
k=k_{0}+k_{1} q+\ldots+k_{w} q^{w} \quad\left(0 \leq k_{i}<q\right)
$$

we put

$$
\mathrm{D}_{k}(x)=\prod_{n=0}^{w} \mathcal{D}_{n}^{k_{n}}(x), \quad k \geq 1 ; \quad \mathrm{D}_{0}(x)=1 .
$$

On applying the digit principle to $\left\{\mathcal{D}_{n}(x)\right\}_{n \geq 0}$ we have

THEOREM 2.1. $\left\{\mathrm{D}_{k}(x)\right\}_{k \geq 0} \quad$ is an orthonormal basis of the space $C\left(\mathbb{F}_{q}[[T]], \mathbb{F}_{q}((T))\right)$.

By reformulating Serre's Lemme I (see [Se]) Conrad gave a nice and simple proof of Theorem 2.1 but did not derive an explicit formula which recovers the expansion coefficients when any function $f \in C\left(\mathbb{F}_{q}[[T]], \mathbb{F}_{q}((T))\right)$ is written uniquely as $f=\sum_{k=0}^{\infty} B_{k} \mathrm{D}_{k}(x)$ where $B_{k} \in \mathbb{F}_{q}((T))$ tend to 0 as $k \rightarrow \infty$. We will focus on deriving a formula for expansion coefficients even though it was already discovered by the author in indirect ways (see $[\mathrm{J} 1, \mathrm{~J} 2])$. The derivation we will give here is intrinsic and natural in the sense that it follows from the proof of Theorem 2.1 together with the basic properties of $\mathrm{D}_{k}^{*}$ defined below. Moreover, we shall derive some interesting results parallel to those known for the Carlitz polynomials in [C2], in terms of the digit derivatives.

The main ingredient of the digit principle (for proving Theorem 2.1) is to show that for any integer $m \geq 0$ the reduced maps $\overline{\mathrm{D}}_{0}, \overline{\mathrm{D}}_{1}, \ldots, \overline{\mathrm{D}}_{q^{m}-1}$ span $\operatorname{Maps}\left(\mathbb{F}_{q}[[T]] / T^{m}, \mathbb{F}_{q}\right)$ as an $\mathbb{F}_{q}$-vector space, where $\operatorname{Maps}(X, Y)$ denotes the 
set of maps from $X$ to $Y$. For cardinality reasons, this is equivalent to showing that they are linearly independent in $\operatorname{Maps}\left(\mathbb{F}_{q}[[T]] / T^{m}, \mathbb{F}_{q}\right)$. Another remark to Theorem 2.1 is that the set of all possible finite $\mathbb{F}_{q}((T))$-linear combinations of $\mathrm{D}_{k}$ 's, denoted $\operatorname{Span}_{\mathbb{F}_{q}((T))}\left\{\mathrm{D}_{i}: i \geq 0\right\}$, is dense in the entire space $C\left(\mathbb{F}_{q}[[T]], \mathbb{F}_{q}((T))\right)$.

Unlike the Carlitz polynomials, we are dealing with non-polynomial functions, so it is necessary to define an order associated to a function $f \in \operatorname{Span}_{\mathbb{F}_{q}((T))}\left\{\mathrm{D}_{i}: i \geq 0\right\}$, which corresponds to the degree of a polynomial. We say that $f$ is of order $d$, denoted $\operatorname{ord}(f)$, if $d$ is the maximum of those $i$ with $B_{i} \neq 0$ in the expansion of $f$ of the form $f(x)=\sum_{i=0}^{k} B_{i} \mathrm{D}_{i}(x)$. We now define $\mathrm{D}_{k}^{*}$, as a variant of $\mathrm{D}_{k}$, in such a way that Carlitz [C2] defined his polynomials $G_{k}^{*}$. For an integer $k \geq 0$ given in (3), set

$$
\mathrm{D}_{k}^{*}(x)=\prod_{n=0}^{w} \mathrm{D}_{k_{n} q^{n}}^{*}(x),
$$

where

$$
\mathrm{D}_{c q^{n}}^{*}(x)= \begin{cases}\mathcal{D}_{n}^{c}(x) & \text { if } 0 \leq c<q-1, \\ \mathcal{D}_{n}^{c}(x)-1 & \text { if } c=q-1 .\end{cases}
$$

Observe that $\mathrm{D}_{q^{n}}(x)=\mathrm{D}_{q^{n}}^{*}(x)=\mathcal{D}_{n}(x), \mathrm{D}_{k}(x)$ and $\mathrm{D}_{k}^{*}(x)$ have order $k$. Note that all non-zero polynomials in $T$ of degree $<m$ are killed by $\mathrm{D}_{q^{m}-1}^{*}(x)$ but $\mathrm{D}_{q^{m}-1}^{*}(0)=(-1)^{m}$. Since the digit derivatives $\mathrm{D}_{k}(x)$ and Carlitz polynomials $G_{k}(x)$ are $q$-adic extensions of hyperdifferential operators $\mathcal{D}_{n}(x)$ and (normalized) Carlitz linear polynomials $E_{n}(x)$ respectively, via the digit expansion, $\mathrm{D}_{k}(x)$ have the same properties with $G_{k}(x)$ such as the binomial formula. For later use we state the binomial formula for $\mathrm{D}_{k}$ and $\mathrm{D}_{k}^{*}$.

Lemma 2.2 ([C2, J1]). (1) $\mathrm{D}_{k}(x+u)=\sum_{i+j=k}\left(\begin{array}{c}k \\ i\end{array}\right) \mathrm{D}_{i}(x) \mathrm{D}_{j}(u)$.

(2) $\mathrm{D}_{k}(x-u)=\sum_{i+j=k}(-1)^{j}\left(\begin{array}{c}k \\ i\end{array}\right) \mathrm{D}_{i}(x) \mathrm{D}_{j}(u)$.

(3) $\mathrm{D}_{k}^{*}(x+u)=\sum_{i+j=k}\left(\begin{array}{c}k \\ i\end{array}\right) \mathrm{D}_{i}(x) \mathrm{D}_{j}^{*}(u)$.

(4) $\mathrm{D}_{k}^{*}(x-u)=\sum_{i+j=k}(-1)^{j}\left(\begin{array}{c}k \\ i\end{array}\right) \mathrm{D}_{i}(x) \mathrm{D}_{j}^{*}(u)$.

We now deal with interpolation series of continuous functions in the space $C\left(\mathbb{F}_{q}[[T]], \mathbb{F}_{q}((T))\right)$ in terms of $\mathrm{D}_{k}^{*}(x)$. For $k$ given in (3), write

$$
\mathrm{D}_{k}^{*}(x)=\left(\mathcal{D}_{0}^{k_{0}}(x)-\delta_{k_{0}(q-1)}\right) \ldots\left(\mathcal{D}_{w}^{k_{w}}(x)-\delta_{k_{w}(q-1)}\right),
$$

where $\delta_{i j}$ is the Kronecker delta. Expanding out the right hand side, we get

$$
\mathrm{D}_{k}^{*}(x)=\mathrm{D}_{k}(x)+\sum_{i=0}^{k-1} C_{i, k} \mathrm{D}_{i}(x),
$$

where $C_{i, k} \in\{-1,0,1\}$. Thus the transition matrix of $\left\{\mathrm{D}_{i}^{*}(x): 0 \leq i \leq k\right\}$ to $\left\{\mathrm{D}_{i}(x): 0 \leq i \leq k\right\}$ is a lower triangular matrix with diagonal entries all 1 , so Serre's lemma and Theorem 2.1 give 
Corollary 2.3. $\left\{\mathrm{D}_{k}^{*}(x)\right\}_{k \geq 0}$ is an orthonormal basis of the space $C\left(\mathbb{F}_{q}[[T]], \mathbb{F}_{q}((T))\right)$.

We remark that by the same argument above the collection of a variant of Carlitz (normalized) polynomials $G_{k}^{*}$ given in $[\mathrm{C} 2, \mathrm{Y} 2, \mathrm{~J} 1]$ is also an orthonormal basis of $C\left(\mathbb{F}_{q}[[T]], \mathbb{F}_{q}((T))\right)$. Unlike Theorem 2.12 below there is no known formula for expansion coefficients in a non-trivial infinite representation of a function $\in C\left(\mathbb{F}_{q}[[T]], \mathbb{F}_{q}((T))\right)$ in terms of $\mathrm{D}_{k}^{*}$, nor is there one for $G_{k}^{*}$.

Let $f(x)$ be a function in $\operatorname{Span}_{\mathbb{F}_{q}((T))}\left\{\mathrm{D}_{i}: i \geq 0\right\}$ of order $k<q^{m}$, then it can be uniquely written as

$$
f(x)=B_{0}+B_{1} \mathrm{D}_{1}(x)+\ldots+B_{k} \mathrm{D}_{k}(x) .
$$

As Carlitz did, we are in a position to recover the coefficients $B_{i}$. For this we need first two crucial lemmas.

LEMMA 2.4. Let $f(x)$ be a function in $\operatorname{Span}_{\mathbb{F}_{q}((T))}\left\{\mathrm{D}_{i}: i \geq 0\right\}$ of order $\leq k<q^{m}$ for some integer $m \geq 0$. If $f(a)=0$ for all a in $\mathbf{A}$ of degree $<m$, then $f$ is identically zero.

Proof. Write $f$ as in (4). Suppose $f$ is not identically zero. Then $f$ can be scaled so that $\|f\|=\max _{0 \leq i \leq k}\left\{\left|B_{i}\right|\right\}=1$. This implies that all $B_{i}$ lie in $\mathbb{F}_{q}[[T]]$ and $B_{j}$ does not belong to $(T)$ for some $0 \leq j \leq k$. Now taking the reduction of $f$ modulo $(T)$ gives

$$
\bar{f}=\sum_{i=0}^{k} \bar{B}_{i} \overline{\mathrm{D}}_{i}
$$

Since $1, T, \ldots, T^{m-1}$ span $\mathbb{F}_{q}[[T]] / T^{m}$ as an $\mathbb{F}_{q}$-vector space, by hypothesis, $\bar{f}$ is a zero map viewed as an element in $\operatorname{Maps}\left(\mathbb{F}_{q}[[T]] / T^{m}, \mathbb{F}_{q}\right)$. As the reduced maps $\overline{\mathrm{D}}_{i}$ are linearly independent over $\mathbb{F}_{q}$ from the remark to Theorem 2.1, all $\bar{B}_{i}$ are 0 , implying that all $B_{i}$ belong to $(T)$. This is a contradiction.

LEMMA 2.5. Let $f(x)$ be a function in $\operatorname{Span}_{\mathbb{F}_{q}((T))}\left\{\mathrm{D}_{i}: i \geq 0\right\}$ of order $\leq k<q^{m}$ for some integer $m \geq 0$. If $f(a)=0$ for all monics a in $\mathbf{A}$ of degree $m$, then $f$ is identically zero.

Proof. The proof is exactly the same as above since the reduced elements of monics in $\mathbf{A}$ of degree $m$ span $\mathbb{F}_{q}[[T]] / T^{m}$ as an $\mathbb{F}_{q}$-vector space.

Lemmas 2.4 and 2.5 are analogous to polynomial situations and they hold for any integer $m$ such that $q^{m}>\operatorname{ord}(f)$.

Let us consider an auxiliary function associated to $f$ in (4):

$$
\Phi(x)=\sum_{\substack{a \in \mathbf{A} \\ \operatorname{deg}_{T}(a)<m}} f(a) \mathrm{D}_{q^{m}-1}^{*}(x-a),
$$


where $q^{m}>k=\operatorname{ord}(f)$. The order of $\Phi(x)$ is at most $q^{m}-1$. If now $a$ is any polynomial in $T$ of degree $<m$, then it is easily seen that (5) gives

$$
\Phi(a)=(-1)^{m} f(a) .
$$

Therefore, it follows from Lemma 2.4 that

$$
\Phi(x)=(-1)^{m} f(x) .
$$

On the other hand, by Lemma 2.2(4) applied to $k=q^{m}-1$, rewrite (5) as

$$
\Phi(x)=\sum_{i+j=q^{m}-1} \mathrm{D}_{i}(x) \sum_{\substack{a \in \mathbf{A} \\ \operatorname{deg}_{T}(a)<m}} \mathrm{D}_{j}^{*}(a) f(a) .
$$

Comparing this with (6) we have at once

TheOREM 2.6. Let $f=\sum_{i=0}^{k} B_{i} \mathrm{D}_{i}(x)$ be a function of order $k<q^{m}$. Then

$$
B_{i}=(-1)^{m} \sum_{\substack{a \in \mathbf{A} \\ \operatorname{deg}_{T}(a)<m}} \mathrm{D}_{q^{m}-1-i}^{*}(a) f(a) \quad \text { for } i<q^{m} .
$$

LEMMA 2.7. Let $f$ be a function of any order in $\operatorname{Span}_{\mathbb{F}_{q}((T))}\left\{\mathrm{D}_{i}: i \geq 0\right\}$ and $q^{m}, q^{n}>i$. Then

$$
(-1)^{m} \sum_{\substack{a \in \mathbf{A} \\ \operatorname{deg}_{T}(a)<m}} \mathrm{D}_{q^{m}-1-i}^{*}(a) f(a)=(-1)^{n} \sum_{\substack{a \in \mathbf{A} \\ \operatorname{deg}_{T}(a)<n}} \mathrm{D}_{q^{n}-1-i}^{*}(a) f(a) .
$$

Proof. Let $q^{m}>q^{n}>i$. Then by definition

$$
\mathrm{D}_{q^{m}-1-i}^{*}=\mathrm{D}_{q^{m}-q^{n}}^{*} \mathrm{D}_{q^{n}-1-i}^{*}
$$

and note that

$$
\mathrm{D}_{q^{m}-q^{n}}^{*}(a)= \begin{cases}0 & \text { if } n \leq \operatorname{deg}_{T}(a)<m, \\ (-1)^{m-n} & \text { if } \operatorname{deg}_{T}(a)<n .\end{cases}
$$

Then the left hand side of the equation in the statement reduces to the right hand side.

We shall now give a formula for expansion coefficients of the representation of $f \in C\left(\mathbb{F}_{q}[[T]], \mathbb{F}_{q}((T))\right)$ in terms of the digit derivatives $\mathrm{D}_{i}$. By Theorem 2.1 write $f(x)=\sum_{i=0}^{\infty} B_{i} \mathrm{D}_{i}(x)$ and as done by Carlitz, divide $f$ into two sums as follows:

$$
f(x)=\sum_{i<q^{m}} B_{i} \mathrm{D}_{i}(x)+\sum_{i \geq q^{m}} B_{i} \mathrm{D}_{i}(x) .
$$

For $a \in \mathbf{A}$ of degree $<m$ satisfying $q^{m}>i$, we see clearly $f(a)=f_{1}(a)$ where $f_{1}(x)$ is the first sum, which is a function of order $<q^{m}$. By the 
coefficient formula in Theorem 2.6, we get, for $i<q^{m}$,

$$
B_{i}=(-1)^{m} \sum_{\substack{a \in \mathbf{A} \\ \operatorname{deg}_{T}(a)<m}} \mathrm{D}_{q^{m}-1-i}^{*}(a) f_{1}(a)=(-1)^{m} \sum_{\substack{a \in \mathbf{A} \\ \operatorname{deg}_{T}(a)<m}} \mathrm{D}_{q^{m}-1-i}^{*}(a) f(a) .
$$

From this and Lemma 2.7 we have

ThEOREM 2.8. Let $f=\sum_{i \geq 0} B_{i} \mathrm{D}_{i}(x)$ be a representation of $f \in$ $C\left(\mathbb{F}_{q}[[T]], \mathbb{F}_{q}((T))\right)$. Then

$$
B_{i}=(-1)^{m} \sum_{\substack{a \in \mathbf{A} \\ \operatorname{deg}_{T}(a)<m}} \mathrm{D}_{q^{m}-1-i}^{*}(a) f(a)
$$

for any integer $m$ such that $q^{m}>i$.

We also define another function analogous to (5):

$$
\Phi_{1}(x)=\sum_{\substack{a \in \mathbf{A}^{+} \\ \operatorname{deg}_{T}(a)=m}} f(a) \mathrm{D}_{q^{m}-1}^{*}(x-a) .
$$

Then $\Phi_{1}(x)$ is of order $<q^{m}$. If now $a$ is any monic in $T$ of degree $m$, then it is easily seen that (7) implies

$$
\Phi_{1}(a)=(-1)^{m} f(a) .
$$

If we now take $q^{m}>k=\operatorname{ord}(f)$, we deduce at once, by Lemma 2.5 , that

$$
\Phi_{1}(x)=(-1)^{m} f(x) .
$$

By Lemma 2.2(4) again, rewrite (7) as

$$
\Phi_{1}(x)=\sum_{i+j=q^{m}-1} \mathrm{D}_{i}(x) \sum_{\substack{a \in \mathbf{A}^{+} \\ \operatorname{deg}_{T}(a)=m}} \mathrm{D}_{j}^{*}(a) f(a) .
$$

Comparing this with (8) leads to

TheOREM 2.9. Let $f=\sum_{i=0}^{k} B_{i} \mathrm{D}_{i}(x)$ be a function of order $k<q^{m}$. Then

$$
B_{i}=(-1)^{m} \sum_{\substack{a \in \mathbf{A}^{+} \\ \operatorname{deg}_{T}(a)=m}} \mathrm{D}_{q^{m}-1-i}^{*}(a) f(a) \quad \text { for } i<q^{m}
$$

We turn to the orthogonality formula for the digit derivatives whose derivation follows on mimicking the proof of the orthogonality for the Carlitz polynomials ([C2, p. 495]). As a special case, consider now $f(x)=\mathrm{D}_{k}(x u)$. We write

$$
\mathrm{D}_{k}(x u)=\sum_{i=0}^{k} \beta_{i}(u) \mathrm{D}_{i}(x),
$$


where $\beta_{i}(u)$ is a function in $u$. Then by Theorems 2.6 and 2.9 we get, respectively

$$
\beta_{i}(u)=(-1)^{m} \sum_{\substack{a \in \mathbf{A} \\ \operatorname{deg}_{T}(a)<m}} \mathrm{D}_{q^{m-1-i}}^{*}(a) \mathrm{D}_{k}(a u)
$$

and

$$
\beta_{i}(u)=(-1)^{m} \sum_{\substack{a \in \mathbf{A}^{+} \\ \operatorname{deg}_{T}(a)=m}} \mathrm{D}_{q^{m}-1-i}^{*}(a) \mathrm{D}_{k}(a u) \quad\left(q^{m}>k\right) .
$$

Putting $u=1$ in (9), we clearly get

Comparison with (10) leads to

$$
\beta_{i}(1)= \begin{cases}0 & \text { if } i<k \\ 1 & \text { if } i=k\end{cases}
$$

$$
\sum_{\substack{a \in \mathbf{A} \\ \operatorname{deg}_{T}(a)<m}} \mathrm{D}_{q^{m}-1-i}^{*}(a) \mathrm{D}_{k}(a)= \begin{cases}0 & \text { if } i<k, \\ (-1)^{m} & \text { if } i=k,\end{cases}
$$

while (11) gives

$$
\sum_{\substack{a \in \mathbf{A}^{+} \\ \operatorname{deg}_{T}(a)=m}} \mathrm{D}_{q^{m}-1-i}^{*}(a) \mathrm{D}_{k}(a)= \begin{cases}0 & \text { if } i<k \\ (-1)^{m} & \text { if } i=k\end{cases}
$$

provided $q^{m}>k$.

Changing the notation slightly, we get the orthogonality formula for the digit derivatives.

Theorem 2.10. (1) For $l<q^{m}, k \geq 0$,

$$
\sum_{\substack{a \in \mathbf{A} \\ \operatorname{deg}_{T}(a)<m}} \mathrm{D}_{k}(a) \mathrm{D}_{l}^{*}(a)= \begin{cases}0 & \text { if } k+l \neq q^{m}-1 \\ (-1)^{m} & \text { if } k+l=q^{m}-1 .\end{cases}
$$

(2) For $l<q^{m}, k<q^{m}$,

$$
\sum_{\substack{a \in \mathbf{A}^{+} \\ \operatorname{deg}_{T}(a)=m}} \mathrm{D}_{k}(a) \mathrm{D}_{l}^{*}(a)= \begin{cases}0 & \text { if } k+l \neq q^{m}-1 \\ (-1)^{m} & \text { if } k+l=q^{m}-1 .\end{cases}
$$

We now provide an interesting property of the digit derivatives, which is analogous to Theorem 5.8.7 of Goss [Go3] for the Carlitz polynomials. For brevity, $\mathbf{A}(t)$ denotes the set of polynomials in $\mathbf{A}$ of degree $<t$.

TheOREM 2.11. Let $f(x)$ be a function in $\operatorname{Span}_{\mathbb{F}_{q}((T))}\left\{\mathrm{D}_{i}: i \geq 0\right\}$ of order $k$ given by $f(x)=\sum_{i=0}^{k} B_{i} \mathrm{D}_{i}(x)$. Then $f(x)$ is invariant under translations by elements of $\mathbf{A}(t)$ if and only if $B_{i}=0$ for $i \neq 0\left(\bmod q^{t}\right)$. 
Proof. It is easy to see that the "if" part comes from the additivity of the hyperdifferential operators $\mathcal{D}_{i}(x)$.

Conversely, let $i \not \equiv 0\left(\bmod q^{t}\right)$. Choose $m$ so that $q^{m}>i$ and $m>t$. Then $\mathbf{A}(t) \subset \mathbf{A}(m)$. Let $\left\{\alpha_{1}, \ldots, \alpha_{q^{m-t}}\right\}$ be representatives of $\mathbf{A}(m) / \mathbf{A}(t)$. The invariance of $f(x)$ under $x \mapsto x+\beta, \beta \in \mathbf{A}(t)$, gives

$$
(-1)^{m} B_{i}=\sum_{j=0}^{q^{m-t}} f\left(\alpha_{j}\right) \sum_{\beta \in \mathbf{A}(t)} \mathrm{D}_{q^{m}-1-i}^{*}\left(\alpha_{j}+\beta\right) .
$$

By Lemma 2.2(3), the sum equals

$$
\sum_{j=0}^{q^{m-t}} f\left(\alpha_{j}\right) \sum_{\beta \in \mathbf{A}(t)} \sum_{e+f=q^{m}-1-i}\left(\begin{array}{c}
q^{m}-1-i \\
f
\end{array}\right) \mathrm{D}_{e}^{*}\left(\alpha_{j}\right) \mathrm{D}_{f}(\beta) .
$$

From Theorem 2.10, we also see that the sum over $\beta$ vanishes unless $f=$ $q^{t}-1$. But, as $i \not \equiv 0\left(\bmod q^{t}\right)$, we have $\left(\begin{array}{c}q^{m}-1-i \\ q^{t}-1\end{array}\right) \equiv 0(\bmod p)$, from which the result follows.

Parallel to (4) consider the representation of a function $f(x)$ of order $\leq k<q^{m}$ in terms of $\mathrm{D}_{i}^{*}(x)$ :

$$
f(x)=\sum_{i=0}^{k} B_{i}^{*} \mathrm{D}_{i}^{*}(x) .
$$

Via $\Phi(x)$ as in (5), one can use Lemma 2.4 to prove

TheOREM 2.12. Let $f(x)$ be a function of order $\leq k$ given by (12). Then

$$
B_{i}^{*}=(-1)^{m} \sum_{\substack{a \in \mathbf{A} \\ \operatorname{deg}_{T}(a)<m}} \mathrm{D}_{q^{m}-1-i}(a) f(a) \quad \text { for } i<q^{m} .
$$

Again we define $\Phi_{1}(x)$ as in (7) and use Lemma 2.5 to derive a formula for the coefficients.

TheOREM 2.13. Let $f(x)$ be a function of order $\leq k$ given by (12). Then

$$
B_{i}^{*}=(-1)^{m} \sum_{\substack{a \in \mathbf{A}^{+} \\ \operatorname{deg}_{T}(a)=m}} \mathrm{D}_{q^{m}-1-i}(a) f(a) \quad \text { for } i<q^{m} .
$$

3. Application to zeta measures. In this section, we relate integral properties of the digit derivatives to the computation of the divided power series associated to the zeta measure at the primes of degree 1 , in particular $(T)$ in $\mathbf{A}$.

The zeta measure $\mu_{x}$ on $\mathbf{A}_{v}$ defined by (1) is rewritten as

$$
\mu_{x}=\sum_{a \in \mathbf{A}^{+}} x^{-\operatorname{deg}_{T}(a)} \delta_{a}
$$


where $\delta_{a}(t)$ is the Dirac measure concentrated at $a$. Hence, when we take $v=(T)$ and $f_{k}=\mathrm{D}_{k}$, the $k$ th coefficient of the divided power series $f_{\mu_{x}}(z)$ is given by the formula

$$
m_{x}(k)=\sum_{a \in \mathbf{A}^{+}} x^{-\operatorname{deg}_{T}(a)} \mathrm{D}_{k}(a)=\sum_{m=0}^{\infty} x^{-m}\left(\sum_{\substack{a \in \mathbf{A}^{+} \\ \operatorname{deg}_{T}(a)=m}} \mathrm{D}_{k}(a)\right) .
$$

To compute the sum in parentheses we apply Theorem 2.13 to a special case.

ThEOREM 3.1.

$$
\sum_{\substack{a \in \mathbf{A}^{+} \\ \operatorname{deg}_{T}(a)=m}} \mathrm{D}_{k}(a)= \begin{cases}(-1)^{m} & \text { if } k=k_{m} q^{m}+q^{m}-1 \text { for } 0 \leq k_{m} \leq q-1, \\ 0 & \text { otherwise. }\end{cases}
$$

Proof. Write $k$ in base $q$ as $k=k_{0}+k_{1} q+\ldots+k_{w} q^{w}, k_{w} \neq 0$. If $w>m$, then $\mathcal{D}_{w}(x)$ kills any polynomial $a \in \mathbf{A}^{+}$of degree $m$. Hence we need only consider the case where $w \leq m$. For $w \leq m$, write $\mathrm{D}_{k}(x)=$ $\mathcal{D}_{m}^{k_{m}}(x) \mathcal{D}_{m-1}^{k_{m-1}}(x) \ldots \mathcal{D}_{0}^{k_{0}}(x)$ where we may assume that $k_{i}=0$ if $i>w$. If $a \in \mathbf{A}^{+}$is of degree $m$, then $\mathcal{D}_{m}(a)=1$, so $\mathrm{D}_{k}(a)=\mathcal{D}_{m-1}^{k_{m-1}}(a) \ldots \mathcal{D}_{0}^{k_{0}}(a)=$ $\mathrm{D}_{k-k_{m} q^{m}}(a)$. Thus we get

$$
\sum_{\substack{a \in \mathbf{A}^{+} \\ \operatorname{deg}_{T}(a)=m}} \mathrm{D}_{k}(a)=\sum_{\substack{a \in \mathbf{A}^{+} \\ \operatorname{deg}_{T}(a)=m}} \mathrm{D}_{k-k_{m} q^{m}}(a) .
$$

Now as $k-k_{m} q^{m}<q^{m}$, we apply Theorem 2.13 with $f(x)=1$ to get the desired result.

We mention here that Theorem 3.1 was first derived by B. Snyder [Sn] via a highly complicated computation. As a corollary, we obtain $m_{x}(k)$ by regrouping sums by degrees of polynomials $a$ in $\mathbf{A}^{+}$.

Corollary 3.2.

$$
\begin{aligned}
& \sum_{a \in \mathbf{A}^{+}} x^{-\operatorname{deg}_{T}(a)} \mathrm{D}_{k}(a) \\
& \quad= \begin{cases}(-1)^{m} x^{-m} & \text { if } k=k_{m} q^{m}+q^{m}-1 \text { and } 1 \leq k_{m}<q-1, \\
(-1)^{m} x^{-m}\left(1-x^{-1}\right) & \text { if } k=q^{m+1}-1, \\
0 & \text { otherwise. }\end{cases}
\end{aligned}
$$

To sum up, we rewrite the $k$ th coefficient $m_{x}(k)$ for an A-magic number [Go2], which is defined as an integer $k$ of the form

$$
k=k_{\mathbf{A}}(c, m)=c q^{m}+q^{m}-1 \quad \text { for } 0<c<q .
$$

THEOREM 3.3. Let $\sum_{k \geq 0} m_{x}(k) z^{k} / k$ ! be the divided power series associated to $\mu_{x}^{(T)}$ with respect to the digit derivatives $\mathrm{D}_{k}(x)$. 
(1) $m_{x}(k) \neq 0$ if and only if $k$ is an $\mathbf{A}$-magic number.

(2) $m_{x}(0)=1$.

(3) Let $k=k_{\mathbf{A}}(c, m)$ be a positive $\mathbf{A}$-magic number. Then

$$
m_{x}(k)= \begin{cases}(-1)^{m} x^{-m} & \text { if } c<q-1 \\ (-1)^{m}\left(1-x^{-1}\right) x^{-m} & \text { if } c=q-1\end{cases}
$$

For comparison we state the remarkable computation by Thakur [Th] of the divided power series for the Carlitz polynomials $G_{k}(x)$.

ThEOREM 3.4. Let $\sum_{k \geq 0} m_{x}(k) z^{k} / k$ ! be the divided power series associated to $\mu_{x}$ with respect to the Carlitz polynomials $G_{k}(x)$.

(1) $m_{x}(k) \neq 0$ if and only if $k$ is an $\mathbf{A}$-magic number.

(2) $m_{x}(0)=1$.

(3) Let $k=k_{\mathbf{A}}(c, m)$ be a positive $\mathbf{A}$-magic number. Then

$$
m_{x}(k)= \begin{cases}(-1)^{m} x^{-m} & \text { if } c<q-1 \\ (-1)^{m}\left(1-x^{-1}\right) x^{-m} & \text { if } c=q-1\end{cases}
$$

We see there is a remarkable similarity between the two divided power series. As the Carlitz polynomials are a (global) orthonormal basis for all places $v$ of $\mathbf{A}$, in some sense, Thakur's result may give a reasonable guess as to how the computation involving the digit derivatives extends to higher degree primes. Unlike the degree 1 primes, it is impossible to get a closed formula for interpolation in higher degree primes, so that one has to search for a new method to complete the zeta measure computation in the case of higher degree primes. The search for such an idea is left to the readers by putting a right digit derivatives basis for higher degree primes. Let $v$ be a

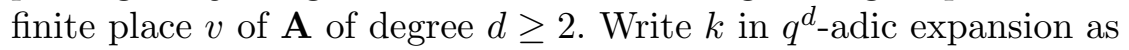

$$
k=k_{0}+k_{1} q^{d}+k_{2} q^{2 d}+\ldots+k_{w} q^{w d}, \quad 0 \leq k_{i}<q^{d} .
$$

With a family of hyperdifferential operators $\mathcal{D}_{n}$, we form the $q^{d}$-adic extensions of $\mathcal{D}_{n}$, which only depend on the degree of the prime:

$$
\mathrm{D}_{k, v}(x)=\mathcal{D}_{0}^{k_{0}}(x) \mathcal{D}_{1}^{k_{1}}(x) \ldots \mathcal{D}_{w}^{k_{w}}(x) .
$$

By Theorem 10 of $[\mathrm{Co}]$, it turns out that this collection of $\mathrm{D}_{k, v}$ is an orthonormal basis of $C\left(\mathbf{A}_{v}, \mathbf{k}_{v}\right)$. Then the computation of the zeta measure at $v$ amounts to the following

Problem. Calculate

$$
\sum_{a \in \mathbf{A}^{+}} x^{-\operatorname{deg}(a)} \mathrm{D}_{k, v}(a)
$$

explicitly for any given integer $k \geq 0$. 
4. Zeta measure of $\mathbb{F}_{q}[T]$ at the infinite place. Recall that $\mathbf{A}=$ $\mathbb{F}_{q}[T], \mathbf{k}=\mathbb{F}_{q}(T), \mathbf{A}_{\infty}=\mathbb{F}_{q}[[\pi]], \mathbf{k}_{\infty}=\mathbb{F}_{q}((\pi))$ and $\pi=1 / T$. We denote by $\widetilde{\mathbf{A}}=\mathbb{F}_{q}[\pi]$ the polynomial ring in $\pi$ over $\mathbb{F}_{q}$ and by $\widetilde{\mathbf{A}}^{+}$the set of monic polynomials in $\pi$ of $\widetilde{\mathbf{A}}$. Then we understand that $\mathbf{A}_{\infty}$ and $\mathbf{k}_{\infty}$ are the completions of $\widetilde{\mathbf{A}}$ and $\mathbf{k}$ at the prime $(\pi)$, respectively. Motivated by D. Thakur's work on the zeta measure at any finite place of $\mathbf{A}$, Z. Yang [Y1, Y2] made an extensive computation of the zeta measure at $\mathbf{A}_{\infty}$ by considering the counterpart $\widetilde{G}_{k}(x)$ of the Carlitz polynomials for the parameter $\pi$. Since the zeta measure at $\pi$ depends only on the choice of an orthonormal basis of $C\left(\mathbf{A}_{\infty}, \mathbf{k}_{\infty}\right)$ it is now natural to ask what is the zeta measure on $\mathbf{A}_{\infty}$ with respect to the digit derivatives basis for the parameter $\pi$. Indeed this basis is formed by the $q$-adic extensions, $\widetilde{\mathrm{D}}_{k}(x)$, of hyperdifferential operators $\widetilde{\mathcal{D}}_{n}$, which are defined by $\widetilde{\mathcal{D}}_{n}\left(\pi^{m}\right)=\left(\begin{array}{c}m \\ n\end{array}\right) \pi^{m-n}$ on $\widetilde{\mathbf{A}}$ for $m \geq 0$. As seen in the previous section, the Carlitz polynomials and digit derivatives share many interesting properties on $\mathbf{A}$, so it is reasonable to expect that such a phenomenon may happen with the computation of the zeta measure at $(\pi)$. Thus we aim to compute the zeta measure at $\mathbf{A}_{\infty}$ with respect to $\widetilde{\mathrm{D}}_{k}(x)$ and compare this with Yang's result. We begin by stating his result:

ThEOREM 4.1. Let $\sum_{k \geq 0} m_{x}(k) z^{k} / k$ ! be the divided power series associated to $\mu_{x}^{(\infty)}$ with respect to the Carlitz polynomials $\widetilde{G}_{k}(x)$.

(1) $m_{x}(0)=1$.

(2) $m_{x}(k)=1$ for $1 \leq k \leq q-2$.

(3) $m_{x}(q-1)=1-\pi^{q-1} x^{-1}$.

(4) If $k=k_{w} q^{w}+\ldots+k_{1} q+k_{0}$ with $k_{w}>0$ and $k \neq q^{w+1}-1$, then take $f \equiv k \bmod (q-1), 0 \leq f<q-1$. Let $k-f=\left(q-1-l_{0}\right)+\left(q-1-l_{1}\right) q+$ $\ldots+\left(q-1-l_{w}\right) q^{w}$. Then

$$
m_{x}(k)=0 \quad \text { if } l_{0}+l_{1}+\ldots+l_{w} \neq q-1
$$

otherwise

$$
\begin{aligned}
m_{x}(k)= & (-1)^{w}\left(\begin{array}{c}
k \\
f
\end{array}\right)\left(\begin{array}{c}
q-1-l_{1} \\
l_{0}
\end{array}\right)\left(\begin{array}{c}
q-1-l_{2} \\
l_{0}+l_{1}
\end{array}\right) \ldots \\
& \times\left(\begin{array}{c}
q-1-l_{w-1} \\
l_{0}+l_{1}+\ldots+l_{w-2}
\end{array}\right) \pi^{\left(\sum_{i=1}^{w} \frac{q^{i}-1}{q-1} l_{i}\right)} x^{-w} .
\end{aligned}
$$

(5) If $k=q^{w+1}-1, w>0$, then

$$
m_{x}(k)=(-1)^{w}\left(1+\pi^{q-1}+\ldots+\pi^{q^{w}-1}\right) x^{-w}+(-1)^{w+1} \pi^{q^{w+1}-1} x^{-(w+1)} .
$$

For a direct comparison with Yang's result, we give the end result of the zeta measure associated to $\widetilde{\mathrm{D}}_{k}(x)$. 
TheOREM 4.2. Let $\sum_{k \geq 0} m_{x}(k) z^{k} / k$ ! be the divided power series associated to $\mu_{x}^{(\infty)}$ with respect to the digit derivatives $\widetilde{\mathrm{D}}_{k}(x)$.

(1) $m_{x}(0)=1$.

(2) $m_{x}(k)=1$ for $1 \leq k \leq q-2$.

(3) $m_{x}(q-1)=1-\pi^{q-1} x^{-1}$.

(4) If $k=k_{w} q^{w}+\ldots+k_{1} q+k_{0}$ with $k_{w}>0$ and $k \neq q^{w+1}-1$, then take $f \equiv k \bmod (q-1), 0 \leq f<q-1$. Let $k-f=\left(q-1-l_{0}\right)+\left(q-1-l_{1}\right) q+$ $\ldots+\left(q-1-l_{w}\right) q^{w}$. Then

$$
m_{x}(k)=0 \quad \text { if } l_{0}+l_{1}+\ldots+l_{w} \neq q-1
$$

otherwise

$$
\begin{aligned}
m_{x}(k)= & (-1)^{w}\left(\begin{array}{c}
k \\
f
\end{array}\right)\left(\begin{array}{c}
q-1-l_{1} \\
l_{0}
\end{array}\right)\left(\begin{array}{c}
q-1-l_{2} \\
l_{0}+l_{1}
\end{array}\right) \ldots \\
& \times\left(\begin{array}{c}
q-1-l_{w-1} \\
l_{0}+l_{1}+\ldots+l_{w-2}
\end{array}\right) \pi^{\left(\sum_{i=1}^{w} i l_{i}\right)} x^{-w} .
\end{aligned}
$$

(5) If $k=q^{w+1}-1, w>0$, then

$$
\begin{aligned}
m_{x}(k)= & (-1)^{w}\left(1+\pi^{q-1}+\pi^{2(q-1)}+\ldots+\pi^{w(q-1)}\right) x^{-w} \\
& +(-1)^{w+1} \pi^{(w+1)(q-1)} x^{-(w+1)} .
\end{aligned}
$$

As mentioned above, it is now not surprising that the two divided power series in Theorems 4.1 and 4.2 have a similar shape but differ slightly only at the exponents of $\pi$. The difference comes from the recursive equations satisfied by the two linear objects, Carlitz linear polynomials and hyperdifferential operators (cf. Lemma 4.3 in [Y2] and Lemma 4.6 below).

The zeta measure $\mu_{x}^{(\infty)}$ defined by formula (2) is reformulated by

$$
\mu_{x}^{(\infty)}(t)=\sum_{a \in \mathbf{A}^{+}} x^{-\operatorname{deg}_{T}(a)} \delta_{\langle a\rangle}(t)
$$

Using this sum we shall completely determine the coefficients of the divided power series associated to the zeta measure on $\mathbf{A}_{\infty}$. As for finite primes, the $k$ th coefficient of the divided power series $f_{\mu_{x}}(z)$ is given by the formula

$$
m_{x}(k)=\sum_{a \in \mathbf{A}^{+}} x^{-\operatorname{deg}(a)} \widetilde{\mathrm{D}}_{k}(\langle a\rangle)=\sum_{j=0}^{\infty} x^{-j}\left(\sum_{\substack{a \in \mathbf{A}^{+} \\ \operatorname{deg}_{T}(a)=j}} \widetilde{\mathrm{D}}_{k}(\langle a\rangle)\right) .
$$

Thus the problem of computing $m_{x}(k)$ is reduced to calculating

$$
\sum_{\substack{a \in \mathbf{A}^{+} \\ \operatorname{deg}_{T}(a)=j}} \widetilde{\mathrm{D}}_{k}(\langle a\rangle)
$$

for a given $k$ and any integer $j \geq 0$. 
For $k=0$ it is easy to see that $m_{x}(0)=1$. By the well known fact (see [C1])

$$
\sum_{\substack{a \in \mathbf{A}^{+} \\ \operatorname{deg}_{T}(a)=j}} a^{k}=0 \quad \text { for } k<q^{j}-1
$$

we easily see that $m_{x}(k)=1$ for $1 \leq k \leq q-2$.

From now on, write $k \neq q-1$ in base $q$ as

$$
k=k_{0}+k_{1} q+\ldots+k_{w} q^{w}, \quad k_{w} \neq 0, w>0 .
$$

To simplify $m_{x}(k)$ we have a simple

LEMMA 4.3. For an integer $k \geq q$ given by (14),

$$
\sum_{\substack{a \in \mathbf{A}^{+} \\ \operatorname{deg}_{T}(a)=j}} \widetilde{\mathrm{D}}_{k}(\langle a\rangle)=0 \quad \text { if } j<w \text { or } j \geq w+2 .
$$

Proof. The case where $j<w$ is clear as $\widetilde{\mathcal{D}}_{w}(x)$ kills polynomials in $\pi$ of degree $<w$. For $j \geq w+2$, write $a=T^{j}+a_{j-1} T^{j-1}+\ldots+a_{1} T+a_{0}$ and $\langle a\rangle=1+a_{j-1} \pi+\ldots+a_{0} \pi^{j}=1+b$ where $b \in W=\operatorname{Span}_{\mathbb{F}_{q}}\left\{\pi, \ldots, \pi^{j}\right\}$. Rewrite and compute the sum as follows:

$$
\sum_{\substack{a \in \mathbf{A}^{+} \\ \operatorname{deg}_{T}(a)=j}} \widetilde{\mathrm{D}}_{k}(\langle a\rangle)=\sum_{b \in W} \widetilde{\mathrm{D}}_{k}(1+b)=\sum_{b \in W} \prod_{i=0}^{w} \widetilde{\mathcal{D}}_{i}(1+b)^{k_{i}}=0,
$$

where the last equality follows from Lemma 8.8.1 of [Go2].

We remark that the sum in Lemma 4.3 also equals 0 if the sum of digits of $k$ is $<(k+1)(q-1)$ even when $\operatorname{dim}_{\mathbb{F}_{q}}(W)=k+1$ in its proof.

By Lemma 4.3, the last equality in (13) reduces to

$$
m_{x}(k)=\left(\sum_{\substack{a \in \mathbf{A}^{+} \\ \operatorname{deg}_{T}(a)=w}} \widetilde{\mathrm{D}}_{k}(\langle a\rangle)\right) x^{-w}+\left(\sum_{\substack{a \in \mathbf{A}^{+} \\ \operatorname{deg}_{T}(a)=w+1}} \widetilde{\mathrm{D}}_{k}(\langle a\rangle)\right) x^{-(w+1)} .
$$

To calculate the two sums in this equation, we state the following result analogous to Theorem 3.1.

LEMMA 4.4 .

$$
\sum_{\substack{a \in \widetilde{\mathbf{A}}^{+} \\ \operatorname{deg}_{\pi}(a)=m}} \widetilde{\mathrm{D}}_{k}(a)= \begin{cases}(-1)^{m} & \text { if } k=k_{m} q^{m}+q^{m}-1 \text { for } 0 \leq k_{m} \leq q-1 \\ 0 & \text { otherwise. }\end{cases}
$$


For $\alpha \in \mathbb{F}_{q}$, set $\mathcal{U}_{\alpha}=\left\{z \in \mathbb{F}_{q}[[\pi]]: z \equiv \alpha \bmod \pi\right\}$, and as the counterpart of $A(d, j, \alpha)=\sum_{a \in \mathcal{U}_{\alpha}, \operatorname{deg}_{\pi}(a)=d} \widetilde{G}_{j}(a)$ defined in [Y2], put

$$
B(d, j, \alpha)=\sum_{\substack{a \in \mathcal{U}_{\alpha} \\ \operatorname{deg}_{\pi}(a)=d}} \widetilde{\mathrm{D}}_{j}(a)
$$

for $\alpha \in \mathbb{F}_{q}$ and $d$ an integer $\geq 0$. Using (15), we rewrite

$$
\sum_{\substack{a \in \mathbf{A}^{+} \\ \operatorname{deg}_{T}(a)=w}} \widetilde{\mathrm{D}}_{k}(\langle a\rangle)=\sum_{d=1}^{w} B(d, k, 1)
$$

Similarly

$$
\sum_{\substack{a \in \mathbf{A}^{+} \\ \operatorname{deg}_{T}(a)=w+1}} \widetilde{\mathrm{D}}_{k}(\langle a\rangle)=\sum_{d=1}^{w+1} B(d, k, 1) .
$$

To simplify the two sums in (16) and (17) we prove the following LEMMA 4.5.

$$
B(d, j, 1)= \begin{cases}B(d, j, 0)+(-1)^{d} & \text { if } j=q^{d+1}-1 \text { or } q^{d}-1 \\ B(d, j, 0) & \text { if } j \equiv 0 \bmod (q-1) \\ & j \neq q^{d+1}-1, j \neq q^{d}-1 .\end{cases}
$$

Proof. This follows from Yang's argument with $A(d, j, 1)$ replaced by $B(d, j, 1)$.

By Lemma 4.5, the computation of $m_{x}(k)$ reduces to two cases depending on the digit expansion of $k$ in (14).

CASE I: $k \neq q^{w+1}-1$ and $w>0$. Then the sum of digits of $k$ is not $(q-1)(w+1)$ so by the remark following Lemma 4.3 and by (16) and (17) it is easily seen that

$$
m_{x}(k)=B(w, k, 1) x^{-w} .
$$

Subcase (a). If $k \not \equiv 0 \bmod (q-1)$, then $k \equiv f \bmod (q-1)$ for some integer $0<f<q-1$. As Yang did, we can deduce the following identity:

$$
B(w, k, 1)=\left(\begin{array}{l}
k \\
f
\end{array}\right) B(w, k-f, 0)
$$

SubCASE (b). If $k \equiv 0 \bmod (q-1)$, then we use Lemma 4.5 to get

$$
B(w, k, 1)=B(w, k, 0) \text {. }
$$

Therefore in either case, we get

$$
m_{x}(k)=B(w, k, 1) x^{-w}=\left(\begin{array}{l}
k \\
f
\end{array}\right) B(w, k-f, 0) x^{-w}
$$

where $f \equiv k \bmod (q-1)$, and $0 \leq f<q-1$. 
CASE II: $k=q^{w+1}-1$. (Here $w=0$ is allowed to fill up the missing case $k=q-1$.) By (16) and (17) we get

$$
m_{x}(k)=B(w, k, 1) x^{-w}+(B(w+1, k, 1)+B(w, k, 1)) x^{-(w+1)} .
$$

Setting $B(0, k, 0)=0$, Lemma 4.5 immediately gives

$$
\begin{aligned}
m_{x}(k)= & \left(B(w, k, 0)+(-1)^{w}\right) x^{-w} \\
& +(B(w+1, k, 0)+B(w, k, 0)) x^{-(w+1)} .
\end{aligned}
$$

From equations (18) and (19), the problem is finally reduced to computing $B(w, k-f, 0), B\left(w, q^{w+1}-1,0\right)$ and $B\left(w+1, q^{w+1}-1,0\right)$. To this end we need to show the following lemma involving $\widetilde{H}_{i}^{s}=\widetilde{\mathcal{D}}_{i}\left(\pi^{i+s}\right)$, which differs from Yang's Lemma 4.3 of [Y2].

LEMma 4.6. The following equation holds:

$$
\widetilde{H}_{w+1}^{0} \pi^{w+1}-\widetilde{H}_{w}^{1} \pi^{w}+\widetilde{H}_{w-1}^{2} \pi^{w-1}+\ldots+(-1)^{w+1} \widetilde{H}_{0}^{w+1} \cdot 1=0 .
$$

Proof. This follows by induction on $w$ using the product rule for $\widetilde{\mathcal{D}}_{i}$ : $\widetilde{\mathcal{D}}_{i}(\pi t)=\pi \widetilde{\mathcal{D}}_{i}(t)+\widetilde{\mathcal{D}}_{i-1}(t)$ for integers $i \geq 1$.

The bulk of remaining computations follows from Yang's derivations with $\widetilde{H}_{i}^{s}=\widetilde{E}_{i}\left(\pi^{i+s}\right)$ replaced by $\widetilde{H}_{i}^{s}=\widetilde{\mathcal{D}}_{i}\left(\pi^{i+s}\right)$, along with Lemma 4.6.

Lemma 4.7. The following formulas hold:

$$
\begin{aligned}
& B\left(w, q^{w+1}-1,0\right)= \begin{cases}(-1)^{w}\left(\pi^{q-1}+\pi^{2(q-1)}+\ldots+\pi^{w(q-1)}\right) & \text { if } w>0, \\
0 & \text { if } w=0 .\end{cases} \\
& B\left(w+1, q^{w+1}-1,0\right)=(-1)^{w+1}\left(\pi^{q-1}+\pi^{2(q-1)}+\ldots+\pi^{(w+1)(q-1)}\right) .
\end{aligned}
$$

Proof. This follows from Lemma 4.4 of [Y2].

It remains to consider the case where $k$ is in Case I. In Subcase (a), if $k$ also satisfies $k-f=f_{w} q^{w}+\ldots+f_{1} q+f_{0}$ and $f_{w}=0$ for some $0<f<q-1$, then it is easy to see that $k-f=q^{w}-1$, and $\left(\begin{array}{c}k \\ f\end{array}\right)=\left(\begin{array}{c}q^{w}+f-1 \\ f\end{array}\right)=0$. Hence by (18) we get $m_{x}(k)=0$. Thus we may assume

$$
k-f=f_{w} q^{w}+\ldots+f_{1} q+f_{0} \quad \text { with } f_{w} \neq 0 \text { in } q \text {-adic expansion, }
$$

and set

$$
l_{i}=q-1-f_{i} \quad \text { for } 0 \leq i \leq w .
$$

Lemma 4.8. If $B(w, k-f, 0) \neq 0$, then $l_{0}+l_{1}+\ldots+l_{w}=q-1$.

Proof. This follows from Lemma 4.6 of [Y2].

Note that Lemma 4.8 also works in the case where $k \equiv 0 \bmod (q-1)$ with $f=0$, which is Subcase (b). We just state $B(w, k-f, 0)$, which follows by imitating the proof of Lemma 4.7 of [Y2], together with Lemmas 4.6 and 4.8 . 
LEMMA 4.9. Let $k-f=f_{w} q^{w}+\ldots+f_{1} q+f_{0}$ with $f_{w} \neq 0$, and let $k-f \equiv 0 \bmod (q-1)$ with $0<f<q-1$ as before. Then $B(w, k-f, 0)$ $=(-1)^{w}\left(\begin{array}{c}q-1-l_{1} \\ l_{0}\end{array}\right)\left(\begin{array}{c}q-1-l_{2} \\ l_{0}+l_{1}\end{array}\right) \cdots\left(\begin{array}{c}q-1-l_{w-1} \\ l_{0}+l_{1}+\ldots+l_{w-2}\end{array}\right) \pi^{l_{1}+2 l_{2}+\ldots+w l_{w}}$. Finally, collecting $m_{x}(k)$ for all $k \geq 0$ yields Theorem 4.2 , as desired.

\section{References}

[C1] L. Carlitz, On certain functions connected with polynomials in a Galois field, Duke Math. J. 1 (1935), 137-168.

[C2] -, A set of polynomials, ibid. 6 (1940), 486-504.

[Co] K. Conrad, The digit principle, J. Number Theory 84 (2000), 230-257.

[Go1] D. Goss, Fourier series, measures and divided power series in the theory of function fields, K-Theory 1 (1989), 533-555.

[Go2] -, Basic Structures of Function Field Arithmetic, Springer, Berlin, 1996.

[Go3] -, L-series of t-motives and Drinfeld modules, in: The Arithmetic of Function Fields, D. Goss et al. (eds.), de Gruyter, 1992, 309-362.

$[\mathrm{H}] \quad$ H. Hasse, Theorie der höheren Differentiale in einem algebraischen Funktionenkörper mit vollkommenem Konstantenkörper bei beliebiger Charakteristik, J. Reine Angew. Math. 175 (1936), 50-54.

[HS] H. Hasse und F. K. Schmidt, Noch eine Begründung der Theorie der höheren Differentialquotienten in einem algebraischen Funktionenkörper einer Unbestimmten, ibid. 177 (1937), 215-237.

[J1] S. Jeong, A comparison of the Carlitz and digit derivatives bases in function field arithmetic, J. Number Theory 84 (2000), 258-275.

[J2] - Hyperdifferential operators and continuous functions on function fields, ibid. 89 (2001), 165-178.

[Se] J.-P. Serre, Endomorphismes complétement continus des espaces de Banach padiques, Inst. Hautes Études Sci. Publ. Math. 12 (1962), 69-85.

[Sn] B. Snyder, Hyperdifferential operators on function fields and their applications, Ph.D. thesis, Ohio State University, Columbus, 1999.

[T] O. Teichmüller, Differentialrechnung bei Charakteristik p, J. Reine Angew. Math. 175 (1936), 89-99.

[Th] D. S. Thakur, Zeta measure associated to $\mathbb{F}_{q}[T]$, J. Number Theory 35 (1990), $1-17$.

[W] C. G. Wagner, Interpolation series for continuous functions on $\pi$-adic completions of $\mathrm{GF}(q, x)$, Acta Arith. 17 (1971), 389-406.

[Y1] Z. Yang, Non-Archimedean analysis over function fields with positive characteristic, Ph.D. thesis, Ohio State University, Columbus, 1999.

[Y2] - A note on zeta measures over function fields, J. Number Theory 90 (2001), 89-112.

Department of Mathematics

Inha University

Incheon, South Korea 402-751

E-mail: stj@inha.ac.kr

Received on 15.2.2002

and in revised form on 11.3.2003 